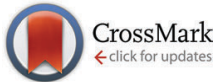

Cite this: Phys. Chem. Chem. Phys., $2016,18,31600$

Received 1st October 2016 Accepted 24th October 2016 DOI: $10.1039 / \mathrm{c} 6 \mathrm{cp} 06749 \mathrm{~b}$

www.rsc.org/pccp

\title{
Self-assembling diacetylene molecules on atomically flat insulators $\dagger$
}

\author{
Elisseos Verveniotis, ${ }^{* a}$ Yuji Okawa, ${ }^{* a}$ Marina V. Makarova, ${ }^{\text {ab }}$ Yasuo Koide, ${ }^{c}$ \\ Jiangwei Liu, ${ }^{C}$ Bretislav Šmíd, ${ }^{\text {cd }}$ Kenji Watanabe, ${ }^{c}$ Takashi Taniguchi, ${ }^{c}$ \\ Katsuyoshi Komatsu, ${ }^{a}$ Takeo Minari, ${ }^{a}$ Xuying Liu, ${ }^{a}{ }^{C}$ Christian Joachim ${ }^{a e}$ and \\ Masakazu Aono ${ }^{a}$
}

\begin{abstract}
Single crystal sapphire and diamond surfaces are used as planar, atomically flat insulating surfaces, for the deposition of the diacetylene compound 10,12-nonacosadiynoic acid. The surface assembly is compared with results on hexagonal boron nitride (h-BN), highly oriented pyrolytic graphite (HOPG) and $\mathrm{MoS}_{2}$ surfaces. A perfectly flat-lying monolayer of 10,12-nonacosadiynoic acid self-assembles on h-BN like on HOPG and $\mathrm{MoS}_{2}$. On sapphire and oxidized diamond surfaces, we observed assemblies of standing-up molecular layers. Surface assembly is driven by surface electrostatic dipoles. Surface polarity is partially controlled using a hydrogenated diamond surface or totally screened by the deposition of a graphene layer on the sapphire surface. This results in a perfectly flat and organized SAM on graphene, which is ready for on-surface polymerization of long and isolated molecular wires under ambient conditions.
\end{abstract}

\section{Introduction}

Progress in electronic nano-device miniaturization attracted considerable interest for single conductive molecular chains ${ }^{1-6}$ which are already utilized for transport measurements and are expected to be integral in the fabrication of molecular electronic devices. $^{5-10}$ On-surface synthesis of molecular chains has been recently performed on clean metallic surfaces in an ultra-high vacuum and at low temperature. ${ }^{11}$ This refreshed the interest for self-assembled monolayers (SAMs) of diacetylene compounds ( $\mathrm{R}-\mathrm{C} \equiv \mathrm{C}-\mathrm{C} \equiv \mathrm{C}-\mathrm{R}^{\prime}$ where $\mathrm{R}$ and $\mathrm{R}^{\prime}$ are substituent groups) because in this case on-surface synthesis of long isolated polydiacetylene (PDA) wires ${ }^{12}\left(=\mathrm{RC}-\mathrm{C} \equiv \mathrm{C}-\mathrm{CR}^{\prime}=\right)_{n}{ }^{5,13,14}$ conveniently

\footnotetext{
${ }^{a}$ International Center for Materials Nanoarchitectonics (WPI-MANA), National Institute for Materials Science (NIMS), 1-1 Namiki, Tsukuba, Ibaraki305-0044, Japan. E-mail: Verveniotis.Elisseos@nims.go.jp, Okawa.Yuji@nims.go.jp

${ }^{b}$ Institute of Physics, Czech Academy of Sciences, Na Slovance, 2, Prague 8, 18221, Czech Republic

${ }^{c}$ National Institute for Materials Science (NIMS), 1-1 Namiki, Tsukuba, Ibaraki 305-0044, Japan

${ }^{d}$ International Institute for Carbon-Neutral Energy Research (WPI- ${ }^{2}$ CNER), Kyushu University, Nishi-ku, Fukuoka 819-0395, Japan

${ }^{e}$ Centre d'Elaboration de Matériaux et d'Études Structurales (CEMES), Centre National de la Recherche Scientifique (CNRS), 29 rue J. Marvig, 31055 Toulouse Cedex, France

$\dagger$ Electronic supplementary information (ESI) available: XPS experimental details and spectra, $I / V$ characteristics of diamond, droplet contact angle measurements, supplementary AFM data and secondary discussion on molecular stacking. See DOI: $10.1039 / \mathrm{c} 6 \mathrm{cp} 06749 \mathrm{~b}$
}

occurs under ambient conditions. ${ }^{5,15}$ Here, substrate heating, ultraviolet (UV) light irradiation or electron tunneling from the tip apex of a scanning tunneling microscope (STM) can initiate the surface topo-chemical chain reaction in air.

An atomically flat supporting surface is an absolute pre-requisite for on-surface synthesis because both the precursor deposition and the subsequent surface chain polymerization are very sensitive to any atomic scale defects even in air: a mono-atomic step edge is enough to terminate chain propagation. ${ }^{16}$ For long PDA chains, $\mathrm{Au}(111),{ }^{17}$ graphite ${ }^{5,18}$ and $\mathrm{MoS}_{2}{ }^{5,14}$ have been reported to provide a very good SAM for STM polymerization. However, single PDA conductance measurements are impossible on those surfaces since the bias voltage applied across a planar junction bridged by a single PDA chain is inherently short-circuited. To avoid this, the diacetylene molecules can, for example, be assembled on a flat, insulating surface that is also cleavable in air like HOPG and $\mathrm{MoS}_{2}$. An obvious candidate would be mica (surface root mean square (RMS) roughness $<0.1 \mathrm{~nm}$ ) but diacetylene molecules like 10,12nonacosadiynoic acid $\left(\mathrm{CH}_{3}\left(\mathrm{CH}_{2}\right)_{15}-\mathrm{C} \equiv \mathrm{C}-\mathrm{C} \equiv \mathrm{C}-\left(\mathrm{CH}_{2}\right)_{8} \mathrm{COOH}\right)$ are reported to be standing-up on it. ${ }^{19}$ Other widely used resistive surfaces like $\mathrm{SiO}_{2}{ }^{20}$ and oxidized $\mathrm{GaN},(\mathrm{Al}, \mathrm{Ga}) \mathrm{N}^{21}$ are too rough for self-assembling flat-lying monolayers of such molecules $(\mathrm{RMS}>0.2 \mathrm{~nm})$.

In this paper, we report and compare the self-assembling of the diacetylene compound 10,12-nonacosadiynoic acid on different atomically flat insulators in air: hexagonal boron nitride (h-BN) on $\mathrm{SiO}_{2}$, polished sapphire and oxidized, highly polished monocrystalline diamond (O-MCD). To obtain further insights into the assembly mechanism we also use hydrogenated MCD (H-MCD). 
Finally, a graphene monolayer was deposited on sapphire in order to screen the long range interactions between the diacetylene molecules and the sapphire surface, resulting in a flat-lying SAM. We propose the usage of functionalized graphene-on-sapphire for future single PDA transport measurements.

\section{Materials and methods}

Atomic force microscopy (AFM) experiments were performed using a Keysight 5500 system (Keysight Co, Santa Rosa, CA, USA) and Multi75-G silicon cantilevers (Budget sensors, Sofia, Bulgaria) with a nominal spring constant and a resonance frequency of $3 \mathrm{Nm}^{-1}$ and $75 \mathrm{kHz}$, respectively. We used both tapping (TM) and contact mode (CM) of operation. All presented AFM images were recorded in TM. Relative humidity and temperature during all AFM experiments were in the ranges of $35-50 \%$ and $22-26{ }^{\circ} \mathrm{C}$, respectively.

h-BN nanosheets of several 10s of $\mu \mathrm{m}$ in lateral size were exfoliated from ultrapure h-BN single crystals ${ }^{22}$ onto $13 \times 13 \mathrm{~mm}$ $\mathrm{Si} / \mathrm{SiO}_{2}$ samples. As optical contrast of the h-BN flakes on $\mathrm{SiO}_{2}$ is very different when compared to that of the substrate, they are clearly resolvable in a standard optical microscope. Then, the substrates were annealed at $500{ }^{\circ} \mathrm{C}$ for 2 hours in air for removing scotch tape residues and atmosphere adsorbates (h-BN is stable up to $1000{ }^{\circ} \mathrm{C}^{23}$ ).

The $10 \times 10 \mathrm{~mm}$ ultra-polished sapphire substrates (Shinkosha Co., Kanagawa, Japan) exhibited millimeter-long, sub-100 nm wide terraces, separated by atomic steps. They were annealed at $1500{ }^{\circ} \mathrm{C}$ in air before use. Besides the inherent contamination removal, high temperature annealing of sapphire is known to induce surface reconstruction. ${ }^{24}$ This resulted in nearly atomically flat terraces (the typical RMS roughness of a $500 \times 500 \mathrm{~nm}^{2}$ area is sub- $0.07 \mathrm{~nm}$ ) that maintained their pre-annealing length, but were now several 100s of nm wide (Fig. S8 in the ESI $\dagger$ ).

$3 \times 3 \mathrm{~mm}$ intrinsic single crystal diamond substrates (Element Six Co., Luxemburg) were also highly polished (Namiki Co., Tokyo, Japan) to achieve sub-0.1 nm roughness (typical RMS $\sim 0.05 \mathrm{~nm}$ ). Surface oxidation was performed by boiling $\left(300{ }^{\circ} \mathrm{C}\right)$ in a $1: 1$ $\mathrm{HNO}_{3} / \mathrm{H}_{2} \mathrm{SO}_{4}$ solution for $3 \mathrm{~h} .{ }^{25}$ Hydrogenation was performed by heating the substrate at $900{ }^{\circ} \mathrm{C}$ in a radio frequency, $\mathrm{H}$ plasma atmosphere $(900 \mathrm{~W})$ for $1 \mathrm{~h} .{ }^{26}$ Since H-MCD is surfaceconductive $^{27}$ and O-MCD is highly resistive, ${ }^{28}$ effectiveness of diamond surface treatments was checked by applying a voltage between two terminals that were in contact with the surface using a room temperature prober (HiSOL Inc, Tokyo, Japan). The $I / V$ characteristics can be seen in the ESI $\dagger$ (Fig. S7).

Typical pristine h-BN, O- and H-MCD surfaces are presented in the ESI $\dagger$ (Fig. S9). Highly oriented pyrolytic graphite (HOPG) and $\mathrm{MoS}_{2}$ substrates (SPI Co., West Chester, PA, USA) were always cleaved by scotch tape before use to ensure that the resulting surface was defect- and adsorbate-free.

After substrate preparation, the diacetylene molecules were deposited on them by (1) spin-coating at $8000 \mathrm{rpm}$ immediately after dropcasting a $4 \mu \mathrm{l}$ droplet (on h-BN) or (2) dropcasting a $4 \mu \mathrm{l}$ droplet and blow drying by $\mathrm{N}_{2}$ gas (on all other substrates).
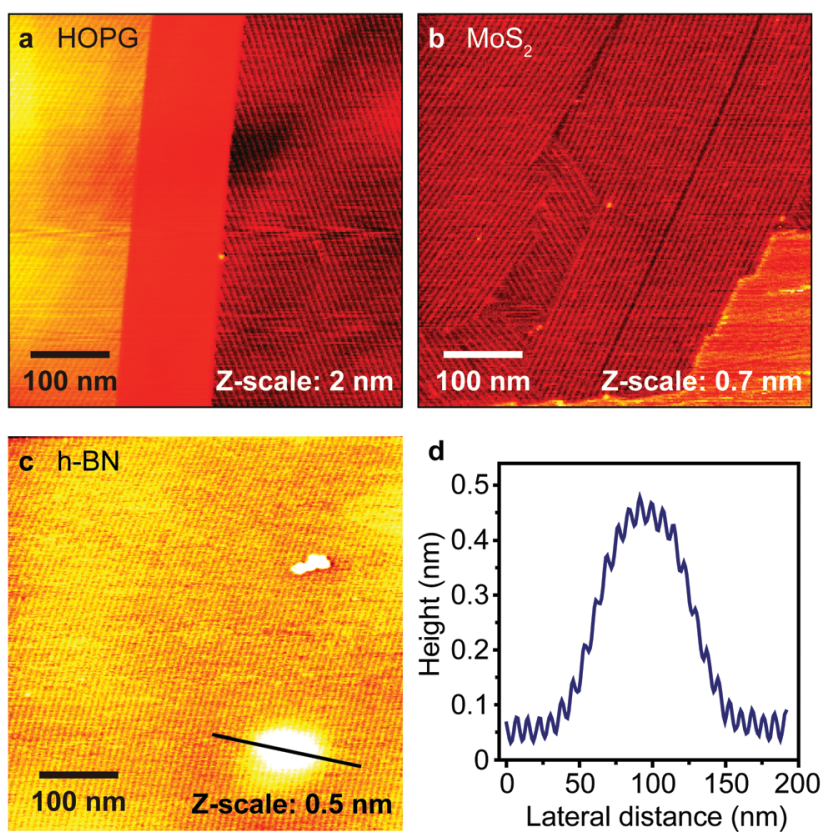

Fig. $1(\mathrm{a}-\mathrm{c})$ AFM topography of the diacetylene molecular layer on HOPG, $\mathrm{MoS}_{2}$ and h-BN, respectively. (d) Spatial profile indicated by the line in (c).

The 10,12-nonacosadiynoic acid diacetylene molecules used here are amphiphilic with a hydrophilic $\mathrm{COOH}$ group at one end and a hydrophobic alkyl chain at the other end. The solution was $0.15 \mathrm{~g} \mathrm{~L}^{-1}$ of 10,12-nonacosadiynoic acid (Tokyo Chemical Industry Co., Ltd) in chloroform. Only in the case of the $\mathrm{SiO}_{2} / \mathrm{h}$-BN system the solvent was xylene due to its lower volatility (the vapor pressure at $20{ }^{\circ} \mathrm{C}$ is $\sim 1330 v s . \sim 21330 \mathrm{~Pa}$ for chloroform) which allowed the performance of the spin-coating process before vaporization of the droplet. The different deposition method used for $\mathrm{SiO}_{2} / \mathrm{h}$-BN was due to the hydrophilicity of the surrounding $\mathrm{SiO}_{2}$. A droplet on such a surface remains on the spot until vaporization/drying (h-BN flakes cover only a small fraction of the sample). This results in large diacetylene aggregates forming on the h-BN flakes within the droplet radius, and no deposition on the flakes outside of it. Note that dropcasting, spin-coating and Langmuir-Schaefer deposition methods yielded similar results in all samples other than $\mathrm{SiO}_{2} / \mathrm{h}-\mathrm{BN}$.

X-ray photoelectron spectroscopy (XPS) was used in order to confirm that the substrates were clean, essentially free of contamination before the diacetylene deposition. The data can be seen in the ESI $\dagger$ (Fig. S1-S6).

\section{Results and discussion}

For reference, Fig. 1a and b show detailed AFM topography $\left(500 \times 500 \mathrm{~nm}^{2}\right)$ after the formation of the 10,12-nonacosadiynoic acid SAM on HOPG and $\mathrm{MoS}_{2}$. The long parallel stripes correspond to self-assembled molecular rows. We can identify domains with different orientation in both cases. In agreement with the literature, ${ }^{5}$ the stripe period is $7.5 \mathrm{~nm}$ on both substrates. The model of molecular arrangement can be seen in ref. 5 . 

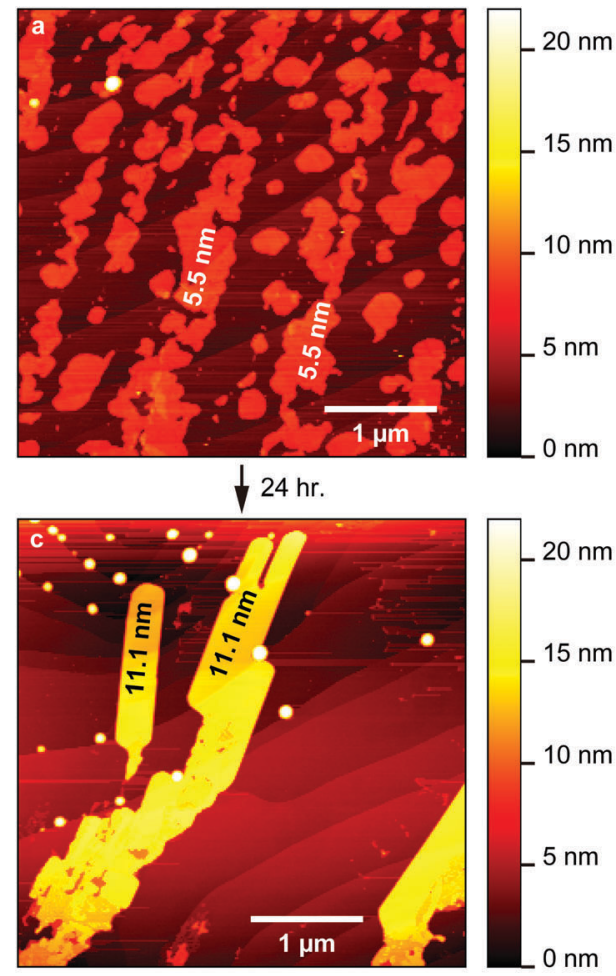

b

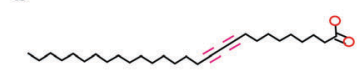

10,12-Nonacosadiynoic acid
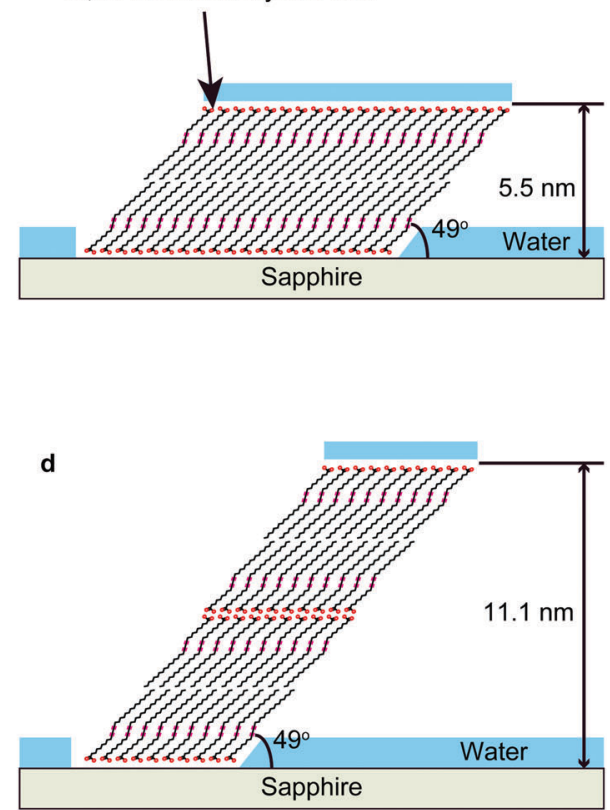

Fig. 2 (a and c) AFM topography of annealed sapphire with dropcast diacetylene immediately after the deposition and $24 \mathrm{~h}$ after it, respectively. (b and d) Schematic models of the molecular assembly on (a) and (c), respectively.

Fig. 1c depicts the molecular layer on $\mathrm{SiO}_{2} / \mathrm{h}-\mathrm{BN}$ (AFM topography, image size $500 \times 500 \mathrm{~nm}^{2}$ ). The arrangement and the stripe period $(7.6 \pm 0.2 \mathrm{~nm})$ are nearly identical to the HOPG and $\mathrm{MoS}_{2}$ cases. Note that here the molecules are assembled in a single domain (typically few $\mu \mathrm{m}$ in size). This might be due to the superior flatness of h-BN over larger areas, which leads to a reduced nucleation density of the diacetylene SAM. ${ }^{29}$ The circular impurity in the 4th quadrant in Fig. 1c is $\sim 100 \mathrm{~nm}$ in diameter. Its height difference $v s$. the surroundings is up to $0.45 \mathrm{~nm}$, evidenced by the spatial profile in Fig. 1d. This impurity can be attributed to the silicon substrate itself as corroborated by the continuous nature of the molecular layer. This is most likely due to the non-abrupt change in the local height, which allowed for the molecular chains to assemble over the impurity. We expect that even in the case of polymerization, the behavior of the PDA chains would be identical, which hints that long range substrate roughness is acceptable for the deposition and polymerization of diacetylene compounds. This is unlike previous studies ${ }^{16}$ indicating that an abrupt atomic step edge is always enough to terminate the process. It is noteworthy that all surfaces depicted in Fig. 1 exhibit hexagonal lattices, indicating that this might be necessary for flat-lying assembly. ${ }^{30}$

On sapphire, Fig. 2a illustrates the AFM topography over a $4 \times 4 \mu \mathrm{m}^{2}$ area immediately after the dropcasting. Several islands of diacetylene cover large areas of the surface. The height of most islands $v s$. the surroundings is $5.5 \pm 1.0 \mathrm{~nm}$, indicating that the layer count in those aggregates is the same. As the length of the diacetylene molecule is estimated to be about $3.7 \mathrm{~nm}$, and given the island homogeneity, these results indicate that the molecules are standing up and form bilayers on the substrate as illustrated in Fig. 2b. Note that even though the islands are clearly composed of two molecular layers, it was often possible to identify the layer orientation in detailed AFM scans (Fig. S10 in the ESI $\dagger$ ). This is in agreement with the literature ${ }^{19}$ where standing up molecules stacked next to each other formed similar patterns. Since the sapphire surface is hydrophilic, the hydrophilic $\mathrm{COOH}$ moiety of the molecule interacts preferably with the substrate and not with the hydrophobic alkyl chain moiety. This is the reason why the standing-up configuration is more stable on sapphire than the flat-lying.

The inter-island space appears mostly unaffected as confirmed by CM scans performed with high contact force (Fig. S11a, ESI $\dagger$ ). The debris spread by the tip within the scanned area is most likely ambient contamination with only a small number of adsorbed molecules. Island formation with relatively clean interspace indicates the attractive molecule-molecule interaction.

Fig. $2 \mathrm{c}$ shows the sample topography $24 \mathrm{~h}$ after dropcasting. The smaller islands merged into larger aggregates whose height $v s$. the substrate is again homogeneous, but doubled to $11.1 \pm 1.0 \mathrm{~nm}$. This is equivalent to 4 standing-up molecular layers, as illustrated in Fig. 2d. The rest of the surface seems to be clean with the sapphire terraces, the steps of which do not seem to influence the molecular assembly, clearly resolvable. Note that further incubation (up to $72 \mathrm{~h}$ ) did not lead to higher aggregations $(6,8,10$ layers, etc.). We can assume that the amount of stable molecular layers is analogous to the solution concentration (higher concentrationmore layers). The molecular deposition on sapphire clearly leads to a Volmer-Weber (island) growth. 

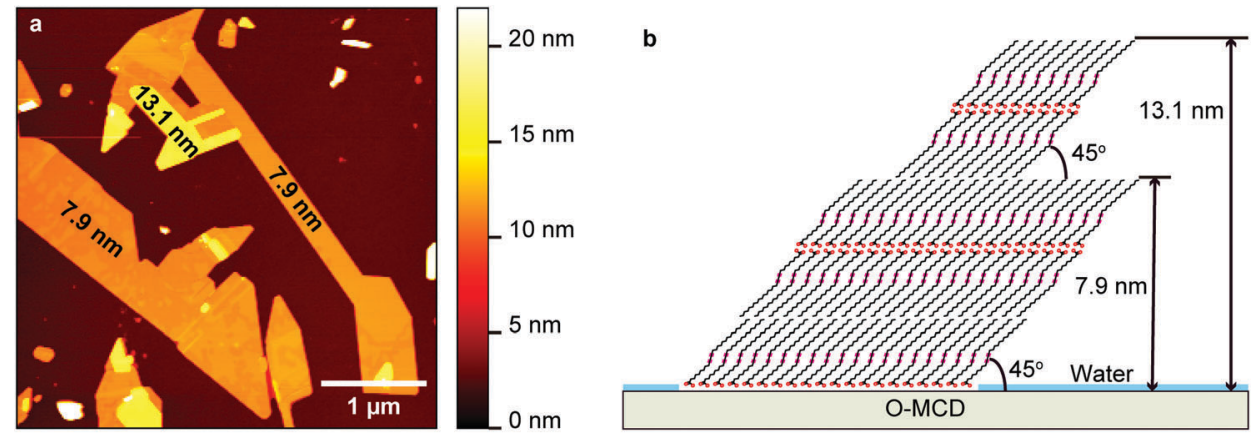

Fig. 3 (a) AFM topography of highly polished oxidized single crystal diamond after dropcasting of diacetylene. (b) Schematic model of the molecular assembly.

After sapphire, oxidized diamond (bandgap typically $5.5 \mathrm{eV}^{31}$ ) was used for the deposition of PDA-precursors. Fig. 3a displays the AFM topography of a $4 \times 4 \mu \mathrm{m}^{2}$ area on the O-MCD substrate immediately after dropcasting the diacetylene solution. Similar to sapphire, islands of diacetylene are formed on the surface. However, this time the islands are $\mu \mathrm{m}$-sized immediately after the deposition. In addition, they are stable as their shape and size do not change with time. The islands have sharp edges, indicating that after the first layer is assembled on the surface, the subsequent molecules adhere while preserving the orientation. The height of those islands is $7.9 \pm 1.0 \mathrm{~nm}$, while there are few areas $13.1 \pm 1.0 \mathrm{~nm}$ above the surface, as noted in the image. Those values give a ratio of $3: 5$ which corresponds to 3 or 5 standing up molecular layers. This configuration is illustrated in Fig. 3b. The inter-island space seems to be clean, similar to sapphire, confirming the attractive molecule-molecule interactions. Growth of diacetylene on O-MCD is therefore also Volmer-Weber.

Sapphire and O-MCD surfaces are hydrophilic and the molecular layers are standing up on them in a similar fashion. However, the stability and the height of the respective molecular islands are different and can be attributed to the higher electronegativity of $\mathrm{C} v s$. $\mathrm{Al}^{32}$ (O has higher electronegativity from both of them). Even though both surfaces are hydrophilic, the $\mathrm{Al}-\mathrm{O}$ surface dipole exhibits a higher negative charge on the $\mathrm{O}$ as compared with the $\mathrm{C}-\mathrm{O}$ dipole. ${ }^{33}$ This results in a weaker interaction of the $\mathrm{COOH}$ moiety with the O-MCD surface when compared to the sapphire, also corroborated by the cleaner surface after a CM scan (Fig. S11b, ESI $\dagger$ ). Another notable difference between the two systems is the even (sapphire) and odd (O-MCD) number of stacked layers. This means that the top side of the layer is hydrophilic $\mathrm{COOH}$ on sapphire and hydrophobic $\mathrm{CH}_{3}$ on $\mathrm{O}-\mathrm{MCD}$, which suggests that, on the former, the molecular layer is covered in water. This difference is also attributed to the stronger $\mathrm{Al}-\mathrm{O}$ surface dipole which attracts a larger amount of water on sapphire (compared to O-MCD), as illustrated in Fig. $2 \mathrm{~b}$ and d.

Regarding the height and the orientation of molecular layers, we assume them to be the same per substrate (e.g. height of top and bottom bilayers on sapphire is the same). Nevertheless, the measured layer height is slightly different between substrates: in the case of sapphire, a monolayer is $2.8 \pm 0.2 \mathrm{~nm}$ high (11.1 nm:4), while on O-MCD this value is $2.6 \pm 0.2 \mathrm{~nm}$
(13.1 nm:5). This indicates that the angle of the molecule with respect to the substrate is approximately $49^{\circ} \pm 4^{\circ}$ on the former and $45^{\circ} \pm 4^{\circ}$ on the latter. The above is corroborated by the AFM height measurements showing nearly identical monolayer height values per substrate on (1) sapphire, where the top and bottom bilayers are both $\sim 5.5 \mathrm{~nm}$ high and (2) O-MCD when comparing the bottom 3-layer with the top 2-layer $(7.9 \mathrm{~nm}$ and $5.2 \mathrm{~nm}$, respectively).

In order to confirm the surface dipole influence on the molecular assembly, the same MCD sample was cleaned with organics and the diacetylene deposition was repeated after surface hydrogenation. The $\mathrm{C}-\mathrm{H}$ surface dipole has positive charge on the $\mathrm{H}$ (due to the lower electronegativity of $\mathrm{H} v s$. C) and the surface is hydrophobic (contact angle measurements on MCD and sapphire can be seen in the ESI, $\dagger$ Fig. S14). Fig. 4a (AFM topography, $600 \times 600 \mathrm{~nm}^{2}$ ) shows diacetylene aggregates assembled on H-MCD after dropcasting. There is no trend concerning the molecular aggregate height (typically around $40 \mathrm{~nm}$ ), unlike on sapphire and O-MCD. Fig. S12a in the ESI $\dagger$ shows a detailed AFM topography image $\left(200 \times 200 \mathrm{~nm}^{2}\right)$ on top of a molecular aggregate. In the corresponding spatial profile (Fig. S12b, ESI $\uparrow$ ) we see a $0.3 \mathrm{~nm}$ height step between adjacent layers, which corresponds to the distance between two flat-lying molecular layers. ${ }^{34,35}$ This clearly indicates that the diacetylene molecules are not standing up but the molecular axis is parallel to the substrate surface. On the hydrophobic H-MCD surface, the alkyl chain moiety of the molecule should interact with the surface stronger than the $\mathrm{COOH}$ moiety. Therefore, the molecules adsorb on H-MCD in a flat-lying manner.

The inter-aggregate area exhibits flatness comparable to that of the substrate and seems mostly free of diacetylene molecules at first glance. However, a $200 \times 200 \mathrm{~nm}^{2} \mathrm{CM}$ scan performed in the area (indicated by the square in Fig. 4a) revealed what was possibly a surface fully covered by several molecular layers after being disrupted by the AFM tip. We can see the surface after the CM scan in Fig. 4b. It is clearly visible that the CM scan induced a large morphology change in the surface, without affecting the surrounding aggregates. This suggests that the molecules that were moved around by the AFM tip immediately merged to form new aggregates. Thus the inter-aggregate area has a layered structure as well (molecules are flat-lying but the layer count is unknown). This is corroborated by the corresponding 

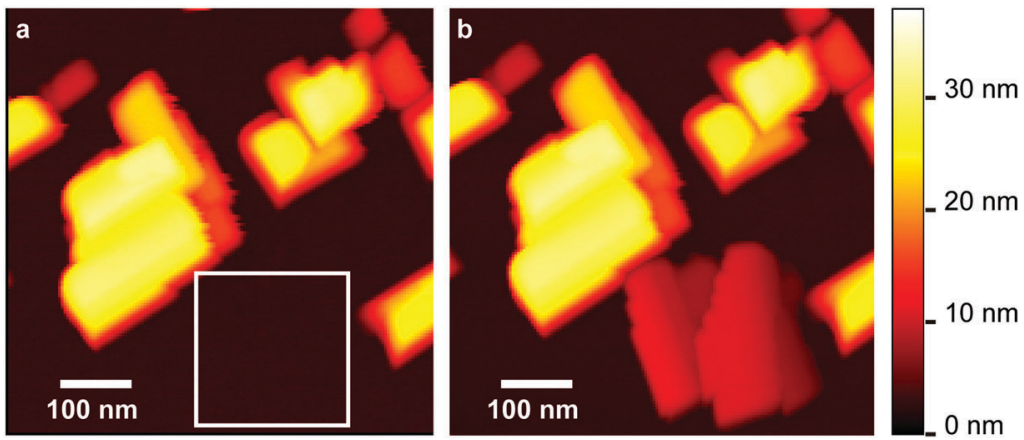

Fig. 4 (a) AFM topography on hydrogenated polished single crystal diamond after dropcasting of diacetylene. (b) AFM topography on the same area as (a), after scanning in contact mode the area indicated by the square in (a).

Table 1 Summarized results

\begin{tabular}{llllll}
\hline Material & Molecular orientation & Layering & Layer height & Monolayer height & Monolayer angle \\
\hline Sapphire & Standing & $2-4$ & $5.5-11.1 \pm 1 \mathrm{~nm}$ & $2.8 \pm 0.2 \mathrm{~nm}$ & $49^{\circ} \pm 4^{\circ}$ \\
O-MCD & Standing & $3-5$ & $7.9-13.1 \pm 1 \mathrm{~nm}$ & $2.6 \pm 0.2 \mathrm{~nm}$ & $45^{\circ} \pm 4^{\circ}$ \\
H-MCD & Flat-lying & Hundreds & Tens of nm & $0.3 \mathrm{~nm}$ & $0^{\circ}$
\end{tabular}

phase shift image (Fig. S13c, ESI $\dagger$ ) which appears homogeneously colored. From Fig. 4a, b and Fig. S13c (ESI $\dagger$ ) it is clear that the growth of diacetylene molecules on H-MCD is Stranski-Krastanov (layer-island), as we can identify both several diacetylene islands of varying height (aggregates) as well as homogeneous layers over larger areas (inter-aggregate space). Note that the molecular structure on H-MCD may be related to the crystalline structure of diacetylene molecules in the bulk phase, which is potentially another indication of strong molecule-molecule interactions. Additional discussion on molecular stacking can be read in the ESI. $\dagger$ All molecular layer data on sapphire and MCD are summarized in Table 1.

Another way to control the hydrophilicity of a surface is by screening the long-range electrostatic interactions at its liquidsurface interface. It was recently demonstrated that only $20 \%$ of those long-range interactions are passing through a monolayer of graphene. ${ }^{36}$ As presented in the optical micrograph in Fig. $5 \mathrm{a}$ a graphene layer was mechanically exfoliated on the ultra-flat sapphire surface before introducing the diacetylene. Fig. 5b depicts AFM topography $\left(500 \times 500 \mathrm{~nm}^{2}\right)$ of diacetylene chains self-assembled on the graphene-on-sapphire system. The planar self-assembly appears now similar to the one observed on HOPG, providing a flat-lying SAM albeit with smaller domains than on HOPG. Graphene adhesion on the sapphire substrate is good as evidenced by the two distinct sapphire terraces that can be seen through the graphene in the AFM topography. The result in Fig. 5 indicates that even though we cannot use sapphire directly for the deposition of the diacetylene molecules, it is an excellent atomically flat candidate for graphene support. The graphene screening effect is ensuring the planar self-assembly of diacetylene in air. As graphene can be rendered insulating, while preserving its flatness, ${ }^{37}$ with $\mathrm{nm}$ precision by functionalizing it with a moderated He-ion beam, ${ }^{38}$ this system can be readily used for on-surface synthesis of single PDA molecular wires and subsequent PDA transport measurements.
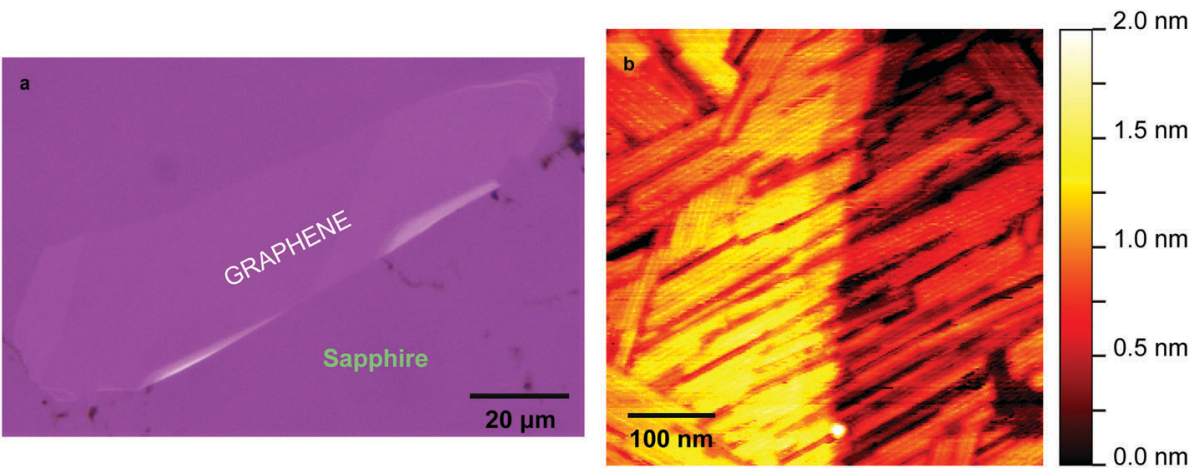

Fig. 5 (a) Optical micrograph $\left(120 \times 80 \mu \mathrm{m}^{2}\right)$ of a graphene flake deposited on an ultra-flat sapphire surface. (b) AFM topography $\left(500 \times 500 \mathrm{~nm}{ }^{2}\right)$ showing diacetylene chains lying flat on the graphene. The sapphire terraces roughly dividing the image into halves indicate good adhesion of the graphene on the substrate. 


\section{Conclusions}

Different substrates were tested to investigate the formation of flat-lying 10,12-nonacosadiynoic acid SAM on a flat insulating surface. The surface of h-BN is appropriate as it provides a SAM similar to HOPG and $\mathrm{MoS}_{2}$, mostly due to its hydrophobic, inert nature and its hexagonal crystal structure. Surface electrostatics (hydrophilicity/hydrophobicity) are essential for the diacetylene arrangement on sapphire, O-MCD and H-MCD. We have demonstrated that sapphire and single crystal diamond are not suitable for self-assembling a flat-lying SAM, as the diacetylene molecules are forming multi-layered aggregates on those substrates. Nevertheless, the polar effect of surfaces like sapphire can be screened by the adsorption of a graphene sheet on which it is possible to form a flat-lying SAM, suitable for on-surface synthesis. The choice between $\mathrm{SiO}_{2} / \mathrm{h}$-BN and graphene-on-sapphire for molecular electronic device fabrication depends on the ability to nanofabricate atomically precise electronic contacts on those surfaces with minimal or no contamination introduced to the system.

\section{Acknowledgements}

We would like to thank Dr M. Osada and Dr S. Nakaharai (NIMS) for important discussions. This work was supported by the World Premier International Research Center Initiative (WPI) and the Ministry of Education, Culture, Sports, Science and Technology of Japan (MEXT), and partially supported by JSPS KAKENHI Grant Numbers 24241047 and 16H03829.

\section{References}

1 N. V. Fischer, U. Mitra, K.-G. Warnick, V. Dremov, M. Stocker, T. Wölfle, W. Hieringer, F. W. Heinemann, N. Burzlaff, A. Görling and P. Müller, Chem. - Eur. J., 2014, 20, 11863-11869.

2 J. Terao, Polym. Chem., 2011, 2, 2444-2452.

3 J. Liu and R. Yang, Phys. Rev. B: Condens. Matter Mater. Phys., 2012, 86, 104307.

4 A. Imamura and Y. Aoki, Int. J. Quantum Chem., 2006, 106, 1924-1933.

5 Y. Okawa, M. Akai-Kasaya, Y. Kuwahara, S. K. Mandal and M. Aono, Nanoscale, 2012, 4, 3013-3028.

6 T. A. Su, H. Li, M. L. Steigerwald, L. Venkataraman and C. Nuckolls, Nat. Chem., 2015, 7, 215-220.

7 H. Song, M. A. Reed and T. Lee, Adv. Mater., 2011, 23, 1583-1608.

8 G. de Ruiter and M. E. van der Boom, J. Mater. Chem., 2011, 21, 17575-17581.

9 N. Fuentes, A. Martín-Lasanta, L. Álvarez de Cienfuegos, M. Ribagorda, A. Parra and J. M. Cuerva, Nanoscale, 2011, 3, 4003-4014.

10 L. Sun, L. Y. A. Diaz-Fernandez, T. A. Gschneidtner, F. Westerlund, S. Lara-Avila and K. Moth-Poulsen, Chem. Soc. Rev., 2014, 43, 7378-7411.

11 C. Nacci, F. Ample, D. Bleger, S. Hecht, C. Joachim and L. Grill, Nat. Commun., 2015, 6, 7397.

12 Y. Okawa and M. Aono, Nature, 2001, 409, 683-684.
13 A. Miura, S. De Feyter, M. M. S. Abdel-Mottaleb, A. Gesquiere, P. C. M. Grim, G. Moessner, M. Sieffert, M. Klapper, K. Mullen and F. C. De Schryver, Langmuir, 2003, 19, 6474-6482.

14 R. Giridharagopal and K. F. Kelly, ACS Nano, 2008, 2, 1571-1580.

15 D. Takajo, A. Inaba and K. Sudoh, Langmuir, 2014, 30, 2738-2744.

16 S. P. Sullivan, A. Schnieders, S. K. Mbugua and T. P. Beebe, Langmuir, 2005, 21, 1322-1327.

17 O. Endo, T. Furuta, H. Ozaki, M. Sonoyama and Y. Mazaki, J. Phys. Chem. B, 2006, 110, 13100-13106.

18 J. P. Rabe, S. Buchholz and L. Askadskaya, Synth. Met., 1993, 54, 339-349.

19 K. Takami, J. Mizuno, M. Akai-Kasaya, A. Saito, M. Aono and Y. Kuwahara, J. Phys. Chem. B, 2004, 108, 16353-16356.

20 D. W. Britt, U. G. Hofmann, D. Moebius and S. W. Hell, Langmuir, 2001, 17, 3757-3765.

21 F. Li, E. Shishkin, M. A. Mastro, J. K. Hite, C. R. Eddy Jr, J. H. Edgar and T. Ito, Langmuir, 2010, 26, 10725-10730.

22 T. Taniguchi and K. Watanabe, J. Cryst. Growth, 2007, 303, 525-529.

23 N. Kostoglou, K. Polychronopoulou and C. Rebholza, Vacuum, 2015, 112, 42-45.

24 K. G. Saw, J. Mater. Sci., 2004, 39, 2911-2914.

25 J. W. Liu, M. Y. Liao, M. Imura, H. Oosato, E. Watanabe, A. Tanaka, H. Iwai and Y. Koide, J. Appl. Phys., 2013, 114, 084108.

26 B. Rezek, L. Michalíková, E. Ukraintsev, A. Kromka and M. Kalbacova, Sensors, 2009, 9, 3549-3562.

27 H. Kawarada, Surf. Sci. Rep., 1996, 26, 205-259.

28 F. Maier, M. Riedel, B. Mantel, J. Ristein and L. Ley, Phys. Rev. Lett., 2000, 85, 3472-3475.

29 R. Xu, D. He, Y. Zhang, B. Wu, F. Liu, L. Meng, J.-F. Liu, Q. Wu, Y. Shi, J. Wang, J.-C. Nie, X. Wang and L. He, Phys. Rev. B: Condens. Matter Mater. Phys., 2014, 90, 224106.

30 M. V. Makarova, Y. Okawa, E. Verveniotis, K. Watanabe, T. Taniguchi, C. Joachim and M. Aono, Nanotechnology, 2016, 27, 395303.

31 E. Verveniotis, J. Čermák, A. Kromka, M. Ledinský and B. Rezek, Nanoscale Res. Lett., 2011, 6, 144.

32 A. L. Allred, J. Inorg. Nucl. Chem., 1961, 17, 215-221.

33 M. Yokoyama and T. Ito, Appl. Surf. Sci., 2000, 161-163, 457-463.

34 P. Nirmalraj, D. Thompson, A. Molina-Ontoria, M. Sousa, N. Martín, B. Gotsmann and H. Riel, Nat. Mater., 2014, 13, 947-953.

35 A. D. Enevoldsen, F. Y. Hansen, A. Diama, L. Criswell and H. Taub, J. Chem. Phys., 2007, 126, 104703.

36 T. Ondarcuhu, V. Thomas, M. Nunez, E. Dujardin, A. Rahman, C. T. Black and A. Checco, Sci. Rep., 2016, 6, 24237.

37 Y. Naitou, T. Iijima and S. Ogawa, Appl. Phys. Lett., 2015, 106, 033103.

38 S. Nakaharai, T. Iijima, S. Ogawa, S. SuzukI, S.-L. Li, K. Tsukagoshi, S. Sato and N. Yokoyama, ACS Nano, 2013, 7, 5694-5700. 